\begin{tabular}{|c|l|}
\hline Title & PDGFRß expression in tumor stroma of pancreatic adenocarcinoma as a reliable prognostic marker \\
\hline Author(s) & Yuzawa, Sayaka; Kano, Mitsunobu R.; Einama, Takahiro; Nishihara, Hiroshi \\
\hline Citation & $\begin{array}{l}\text { Medical Oncology, 29(4), 2824_2830 } \\
\text { https://doi.org/10.1007/312032-012-0193-0 }\end{array}$ \\
\hline Issue Date & 2012-12 \\
\hline Doc URL & http://hdl.handle.net/2115/50788 \\
\hline Rights & The final publication is available at www.springerlink.com \\
\hline Type & article (author version) \\
\hline File Information & Mo29-4_2824_2830.pdf \\
\hline
\end{tabular}

Instructions for use 


\section{PDGFRß expression in tumor stroma of pancreatic adenocarcinoma as a reliable}

\section{prognostic marker}

Sayaka Yuzawa ${ }^{1}$, Mitsunobu R. Kano ${ }^{2, *}$, Takahiro Einama ${ }^{3}$, and Hiroshi Nishihara ${ }^{1}$

Affiliations and addresses:

${ }^{1}$ Department of Translational Pathology, Graduate School of Medicine, Hokkaido University, Kita 15,

Nishi 7, Kita-ku, Sapporo, Hokkaido, 060-8638 Japan: ${ }^{2}$ Medical Scientist Training Program, Faculty

of Medicine, University of Tokyo, 7-3-1 Hongo, Bunkyo-ku, Tokyo 113-0033, Japan: and ${ }^{3}$ Division of

Gastroenterological and General Surgery, Department of Surgery, Asahikawa Medical College, 1-1

Midori-ga-oka Higashi 2-jo 1-chome, Asahikawa-shi, Hokkaido, 078-8510 Japan.

*Corresponding author: Mitsunobu R. Kano

E-mail: mikano-tky@umin.net

Tel \& Fax: +81-3-5841-0476 


\section{Abstract}

Pancreatic adenocarcinoma is a lethal disease that often develops a desmoplastic reaction in tumor stroma. In general, desmoplasia is thought to promote tumor growth. However, its molecular pathology and prognostic potential has not been fully investigated. Here we investigate 26 cases of pancreatic ductal adenocarcinoma, and examine the clinicopathological association between survival and expression levels of several molecular markers for stromal cells. These include alpha smooth muscle actin (SMA) and platelet-derived growth factor (PDGF) receptor $\beta$ (PDGFR $\beta$ ). Both are markers of activated fibroblasts or pancreatic stellate cells (PSCs) that play an important role in desmoplasia. The staining patterns of both molecular markers were not uniform so we categorized them into 3 grades (high, middle, and low) according to intensity. Interestingly, Kaplan-Meier analysis revealed that higher expression of PDGFR $\beta$ matched shorter prognosis $(\mathrm{p}=0.0287, \log$-rank test) as well as lymphatic invasion and lymph node metastasis, whereas SMA did not $(\mathrm{p}=0.6122)$. Our results suggest the prognostic potential of cancer stroma via PDGF-B signaling. Regulation of PDGF-B-associated signaling crosstalk between cancer cells and stroma cells, therefore, may indicate a possible therapeutic target for desmoplastic malignant tumors such as pancreatic adenocarcinoma.

Keywords: pancreatic adenocarcinoma, tumor stroma, prognosis, PDGFR $\beta$, SMA 


\section{Introduction}

Pancreatic adenocarcinoma is a lethal disease with a median survival time of approximately

6 months [1], but exactly why the disease is so difficult to treat is not fully understood.

Staging of the cancer, determined by tumor-node-metastasis (TNM) staging system, can be

divided into prognostic subgroups [2], and several markers in tumor cells are also useful $[3,4]$.

Lymph vessel invasion (ly) is useful as a histology marker, but neither intrapancreatic neural invasion (ne) nor blood vessel invasion (v) have proven clinically useful histology signs [5].

Histopathologically, pancreatic adenocarcinoma is often accompanied by a dense desmoplastic reaction [6], and this constitutes the characteristic stromal structure of the cancer. This desmoplasia forms approximately eighty percent of the tumor mass, and is thought to play an active role in carcinogenesis [7]. In human tumors in general, desmoplastic reaction is associated with recruitment and activation of fibroblasts and significant deposition of extracellular matrix (ECM) [8].

Normal fibroblasts, which are embedded in ECM, are activated by various stimuli that accompany insult to tissue. The differentiation between fibroblasts and myofibroblasts in vivo is made by positivity to alpha smooth muscle actin (SMA), and those that are positive are myofibroblasts [9]. Fibroblasts activate and differentiate into the myofibroblast phenotype as part of inflammation, where platelet-derived growth factor (PDGF) induces differentiation $[10,11]$.

The receptor for PDGF-BB, PDGFR $\beta$, has an important role in regulating mesenchymal cells, including pericytes, fibroblasts, and vascular smooth muscle cells, during development [12,13]. 
Activation of PDGFR may be involved in cancer progression via activation of these mesenchymal cells in most solid tumors [14], besides directly stimulating tumor cell growth: as reported for tumors responsive to Imatinib (Gleevec®), an inhibitor of PDGFR [15].

Expression of SMA and PDGFR $\beta$ may thus be important in the myofibroblasts of pancreatic adenocarcinoma stroma. However, their clinical significance has not been investigated in depth. Here we investigate the prognostic potential of tumor stroma markers in pancreatic cancer via immunostaining of SMA and PDGFR $\beta$, and show that expression of PDGFR $\beta$ is the more valuable. 


\section{Materials and Methods}

\section{Patients}

Twenty-six patients with primary pancreatic adenocarcinoma, who underwent pancreaticoduodenectomy (PD) or pylorus-preserving PD (PpPD) in the First Department of Surgery at the Hokkaido University Hospital, Japan, were included in this study. Of the total: 18 were male and 8 female, 14 had adjuvant chemotherapy and 12 did not. The patients ranged in age from 45-81Y. The samples were obtained under blanket, written informed consent, and the experiment approved by the Ethics Committee of Hokkaido University. The clinicopathology of the cases is summarized in Table 1. Pathology staging was made according to the Japan Pancreas Society [16] following TNM classification [17].

\section{Antibodies and Immunohistochemistry}

Tissue sections were prepared from paraffin blocks and stained with hematoxylin and eosin (HE): double immunohistochemical staining was performed using the primary antibodies for PDGFR $\beta$ (CST Japan, Tokyo), or SMA (DAKO Japan Co., Kyoto, Japan) and CD31 (DAKO) followed by detection of the antibody with alkaline phosphatase-conjugated Fast Blue BB / Naphthol AS-MX-phosphate readout system for PDGFR $\beta$ and SMA in red, or a peroxidase-conjugated streptavidin-DAB readout system (DAKO) for CD31 in brown, with nuclear counter staining in blue.

Stain intensity of SMA and PDGFR $\beta$ in cancer stroma was divided into three grades: weak 
(1+), intermediate (2+) and strong (3+). Density of CD31 positive vasculature was evaluated in the same manner as with SMA or PDGFR $\beta$. The histological factors ly (lymphatic invasion), v (vascular invasion), ne (neural invasion) were determined according to routine pathological diagnostic protocol [16].

\section{Statistical Analysis}

$\chi^{2}$ test was used for pre-screening candidate factors, with $\mathrm{p}<0.05$ as statistical significance.

Survival curves were calculated using Kaplan and Meier method, and differences between curves were analyzed using the log-rank test, with $\mathrm{p}<0.05$ as statistical significance. 


\section{Results}

In addition to those factors for routine pathological diagnosis, ly, v, ne, T, N, and M, we divided the stroma samples into three grades (1 to 3) based on immunohistochemistry: that is, positivity for SMA or PDGFR $\beta$, and microvascular density determined by CD31 positive structure. Fig. 1 shows four representative cases with overall survival (OS) in months (m). Expression levels of SMA and PDGFR $\beta$ did not necessarily correlate.

We then compared distribution values for ly, v, ne, T, N, CD31 density, SMA, or PDGFR $\beta$, by $\chi^{2}$ test between patient groups with shorter prognosis than the median period of 15 months $(\mathrm{n}=$ 14), plus the group that survived for more than 15 months $(n=12)$. The results are shown in Figure 2. Analysis revealed that stromal positivity for PDGFR $\beta$ was a factor, with a difference of $p<0.05$ between the two groups, although SMA was not statistically significant. Staging and ly were confirmed to be also statistically significant, as reported previously [2,5]. We also checked for relationship of adjuvant chemotherapy to survival, but there was none (Table 1).

We therefore tested for prognostic impact of PDGFR $\beta$ positivity in stroma in pancreatic adenocarcinoma using Kaplan-Meier survival analysis. Patients strongly positive for PDGFR $\beta$ (3+) were significantly worse off in terms of survival than those with intermediate or weak positivity (2+ and $1+)(p=0.0287$ by log-rank test): Figure 3A. Median survival rates were 22.5 months for lower PDGFR $\beta$ expression and 13.0 months for higher PDGFR $\beta$ expression). On the other hand, strength 
of SMA expression was not significant $(\mathrm{p}=0.6122$ : Figure 3B. Median survival rates were 17.0

months for lower SMA expression and 15.0 months for higher SMA expression. 


\section{Discussion}

Pancreatic adenocarcinoma is a devastating disease characterized by tumor desmoplasia in

stroma: a significant increase in connective tissue that envelopes tumor cell nests [18]. In this patient study we show that more expression of PDGFR $\beta$ in stroma of pancreatic adenocarcinoma correlates with a worse prognosis, while expression levels of SMA do not.

Although the general impact of tumor stroma in pancreatic cancer is poorly understood, there is previous work on stroma as a prognostic marker in pancreatic ductal adenocarcinoma [19]. In this work, ratio of SMA-stained area to collagen-stained area is usefully defined as the "activated stroma index", and a combination of high "stromal activity" and low collagen deposition is linked with a worse prognosis, whereas a combination of high collagen deposition and low stromal activity is linked with a better outcome. However, to our knowledge, there is no report as yet linking PDGFR $\beta$ expression and "stromal activity".

In tumor stroma, desmoplastic reaction is associated with activated fibroblasts positive for SMA, plus significant deposition of ECM including collagen [8]. In the case of pancreatic adenocarcinoma, pancreatic stellate cells (PSCs) are considered another key player in desmoplasia, together with activated myofibroblasts [18,20,21]. Both cells are positive for SMA and collagen [22]. In the pancreas, insult or inflammation stimulates quiescent PSCs to undergo morphological and functional change and become myofibroblast-like cells that express SMA [21]. Pancreatic tumor cells 
induce proliferation of PSCs positive for PDGFR $\beta$, and induce PSC production of ECM proteins via signaling that includes PDGF-BB [23]. PSCs in vitro also increases proliferation, invasion, and colony formation of pancreatic tumor cells, and protect them from attack by radiation and gemcitabine $[24,25]$. Furthermore, they create a tumor-supportive microenvironment by producing ECM [21]. In fact, coinjection of cancer cells with PSCs in vivo increase tumor size in a subcutaneous xenograft model [23] plus tumor incidence, growth, and metastasis in orthotopic models of pancreatic cancer [24,25].

It is interesting, therefore, that SMA does not correlate significantly with prognosis, while PDGFR $\beta$ does, although both SMA and PDGFR $\beta$ are important markers of activated myofibroblasts or PSCs. PDGFR $\beta$ positivity may therefore relate directly to interactive functions of myofibroblasts or PSCs with tumor cells and other stroma cells, while SMA may relate to some autonomous function such as contractile ability. The prognostic impact of PDGFR $\beta$ expression in tumor stroma has been reported for prostate and breast cancer $[12,26]$. PDGF-BB, the ligand of PDGFR $\beta$ functions primarily via paracrine mechanisms involving other cell types, such as fibroblasts and endothelial cells [27]. PDGF-BB induces proliferation of fibroblasts, but does not induce acquisition of an activated phenotype associated with excessive ECM deposition [28]. PDGF-BB are released from injured epithelial cells and infiltrate immune cells as part of the normal wound healing process [29]. Although PDGF-BB is secreted by cancer cells and correlates with cancer progression [30], most cancer cells do not express PDGFR $\beta$. Therefore, it is possible that PDGFR $\beta$ expression in tumor 
stroma may be a universal marker of tumor activity and correlate with disease prognosis.

\section{Acknowledgements}

We are grateful to Prof. Michael W. Miller (Miller Takemoto \& Partners) for help with the manuscript.

This research was supported by a Grant-in-Aid for Scientific Research (KAKENHI) and by the Japan Society for the Promotion of Science (JSPS) through the "Funding Program for World-Leading Innovative R\&D on Science and Technology (FIRST Program)," initiated by the Council for Science and Technology Policy (CSTP). The study sponsors had no involvement in the study design, in the collection, analysis and interpretation of data, in the writing of the manuscript or in the decision to submit the manuscript for publication.

Conflict of Interests: None. 


\section{References}

1. Burris HA, Moore MJ, Andersen J, et al. Improvements in survival and clinical benefit with gemcitabine as first-line therapy for patients with advanced pancreas cancer: a randomized trial. J Clin Oncol. 1997; 15: 2403-13.

2. Katz MHG, Hwang R, Fleming JB, et al. Tumor-node-metastasis staging of pancreatic adenocarcinoma. CA Cancer J Clin. 2008; 58: 111-25.

3. Smith RA, Tang J, Tudur-Smith C, et al. Meta-analysis of immunohistochemical prognostic markers in resected pancreatic cancer. Br J Cancer. 2011; 104: 1440-51.

4. Singh P, Srinivasan R, Wig JD. Major molecular markers in pancreatic ductal adenocarcinoma and their roles in screening, diagnosis, prognosis, and treatment. Pancreas. 2011; 40: 644-52.

5. Mitsunaga S, Hasebe T, Iwasaki M, et al. Important prognostic histological parameters for patients with invasive ductal carcinoma of the pancreas. Cancer Sci. 2005; 96: 858-65.

6. Farrow B, Albo D, Berger DH. The role of the tumor microenvironment in the progression of pancreatic cancer. J Surg Res. 2008; 149: 319-28.

7. Erkan M, Reiser-Erkan C, Michalski CW, et al. Tumor microenvironment and progression of pancreatic cancer. Exp oncol. 2010; 32: 128-31. 
8. Kalluri R, Zeisberg M. Fibroblasts in cancer. Nat Rev Cancer. 2006; 6: 392-401.

9. Watsky MA, Weber KT, Sun Y, et al. New insights into the mechanism of fibroblast to myofibroblast transformation and associated pathologies. Int Rev Cell Mol Biol. 2010; 282: 165-92.

10. Tang WW, Ulich TR, Lacey DL, et al. Platelet-derived growth factor-BB induces renal tubulointerstitial myofibroblast formation and tubulointerstitial fibrosis. Am J Pathol. 1996; 148: 1169-80.

11. Kaur H, Chaurasia SS, Medeiros FW de, et al. Corneal stroma PDGF blockade and myofibroblast development. Exp Eye Res. 2009; 88: 960-5.

12. Paulsson J, Sjöblom T, Micke P, et al. Prognostic significance of stromal platelet-derived growth factor beta-receptor expression in human breast cancer. Am J Pathol. 2009; 175: 334-41.

13. Betsholtz C. Insight into the physiological functions of PDGF through genetic studies in mice. Cytokine Growth Factor Rev. 2004; 15: 215-28.

14. Ostman A, Heldin C-H. PDGF receptors as targets in tumor treatment. Adv Cancer Res. 2007; 97: 247-74. 
15. Apperley JF, Gardembas M, Melo JV, et al. Response to imatinib mesylate in patients with chronic myeloproliferative diseases with rearrangements of the platelet-derived growth factor receptor beta. N Engl J Med. 2002; 347: 481-7.

16. Japan Panceas Society. Classification of Pancreatic Carcinoma. 3rd ed. Tokyo: Kanehara Co.; 2011.

17. AJCC Cancer Staging Manual (Edge, Ajcc Cancer Staging Manual). 7th ed. New York: Springer; 2010.

18. Omary MB, Lugea A, Lowe AW, et al. The pancreatic stellate cell: a star on the rise in pancreatic diseases. J Clin Invest. 2007; 117: 50-9.

19. Erkan M, Michalski CW, Rieder S, et al. The activated stroma index is a novel and independent prognostic marker in pancreatic ductal adenocarcinoma. Clin Gastroenterol Hepatol. 2008; 6: 1155-61.

20. Apte MV, Haber PS, Applegate TL, et al. Periacinar stellate shaped cells in rat pancreas: identification, isolation, and culture. Gut. 1998; 43: 128-33.

21. Masamune A, Watanabe T, Kikuta K, et al. Roles of pancreatic stellate cells in pancreatic inflammation and fibrosis. Clin Gastroenterol Hepatol. 2009; 7: S48-54. 
22. Apte MV, Park S, Phillips PA, et al. Desmoplastic reaction in pancreatic cancer: role of pancreatic stellate cells. Pancreas. 2004; 29: 179-87.

23. Bachem MG, Schünemann M, Ramadani M, et al. Pancreatic carcinoma cells induce fibrosis by stimulating proliferation and matrix synthesis of stellate cells. Gastroenterology. 2005; 128: 907-21.

24. Hwang RF, Moore T, Arumugam T, et al. Cancer-associated stromal fibroblasts promote pancreatic tumor progression. Cancer Res. 2008; 68: 918-26.

25. Vonlaufen A, Joshi S, Qu C, et al. Pancreatic stellate cells: partners in crime with pancreatic cancer cells. Cancer Res. 2008; 68: 2085-93.

26. Hägglöf C, Hammarsten P, Josefsson A, et al. Stromal PDGFRbeta expression in prostate tumors and non-malignant prostate tissue predicts prostate cancer survival. PloS one. 2010; 5: e10747.

27. Forsberg K, Valyi-Nagy I, Heldin CH, et al. Platelet-derived growth factor (PDGF) in oncogenesis: development of a vascular connective tissue stroma in xenotransplanted human melanoma producing PDGF-BB. Proc Natl Acad Sci U S A. 1993; 90: 393-7.

28. Shao ZM, Nguyen M, Barsky SH. Human breast carcinoma desmoplasia is PDGF initiated. Oncogene. 2000; 19: 4337-45. 
29. Zeisberg M, Strutz F, Müller GA. Role of fibroblast activation in inducing interstitial fibrosis. J Nephrol. 13: S111-20.

30. Bronzert DA, Pantazis P, Antoniades HN, et al. Synthesis and secretion of platelet-derived growth factor by human breast cancer cell lines. Proc Natl Acad Sci U S A. 1987; 84: 5763-7. 


\section{Figures legends}

Figure 1. Microphotographs of serial sections of four representative cases, with overall survival (OS) in months (m). Hematoxylin-eosin (HE) staining and immunostaining of alpha-smooth muscle actin (SMA) and platelet-derived growth factor receptor $\beta$ (PDGFR $\beta$ ) (red) together with CD31 staining (brown). Bars: $100 \mu \mathrm{m}$.

Figure 2. Histograms of various clinicopathological factors for two groups of cases divided at the median survival period of 15 months. P values were calculated by $\chi^{2}$ test. $*: p<0.05$.

Figure 3. Kaplan-Meier curves for PDGFR $\beta$ (A) and SMA (B). P values were calculated by log-rank test. $*: \mathrm{p}<0.05$. 
Table 1. The clinicopathology of the cases.

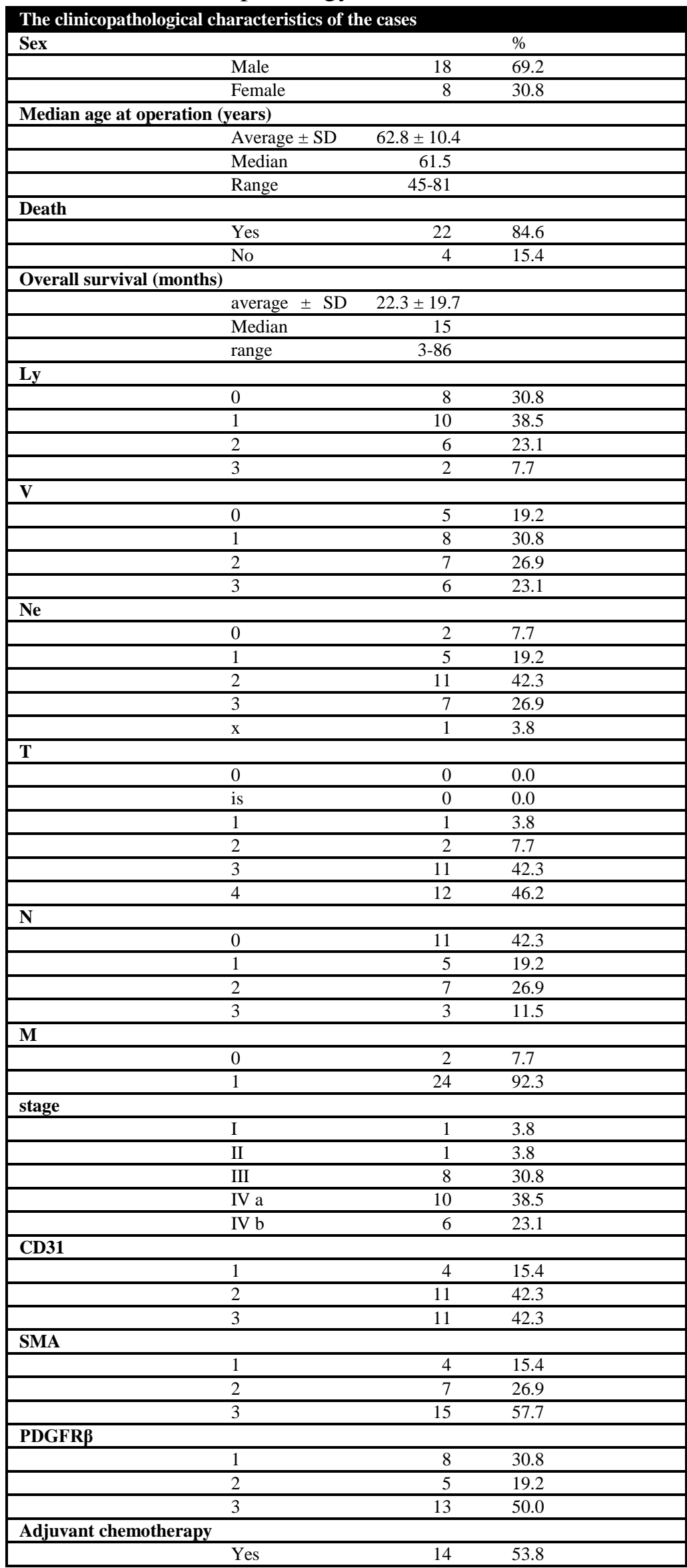




\begin{tabular}{|lcc|}
\hline No & 12 & 46.2 \\
\hline Median survival: $19 \mathrm{~m}$ vs. $14 \mathrm{~m}, \mathrm{p}=0.1351$ (log-rank test). \\
\hline
\end{tabular}


Figure 1

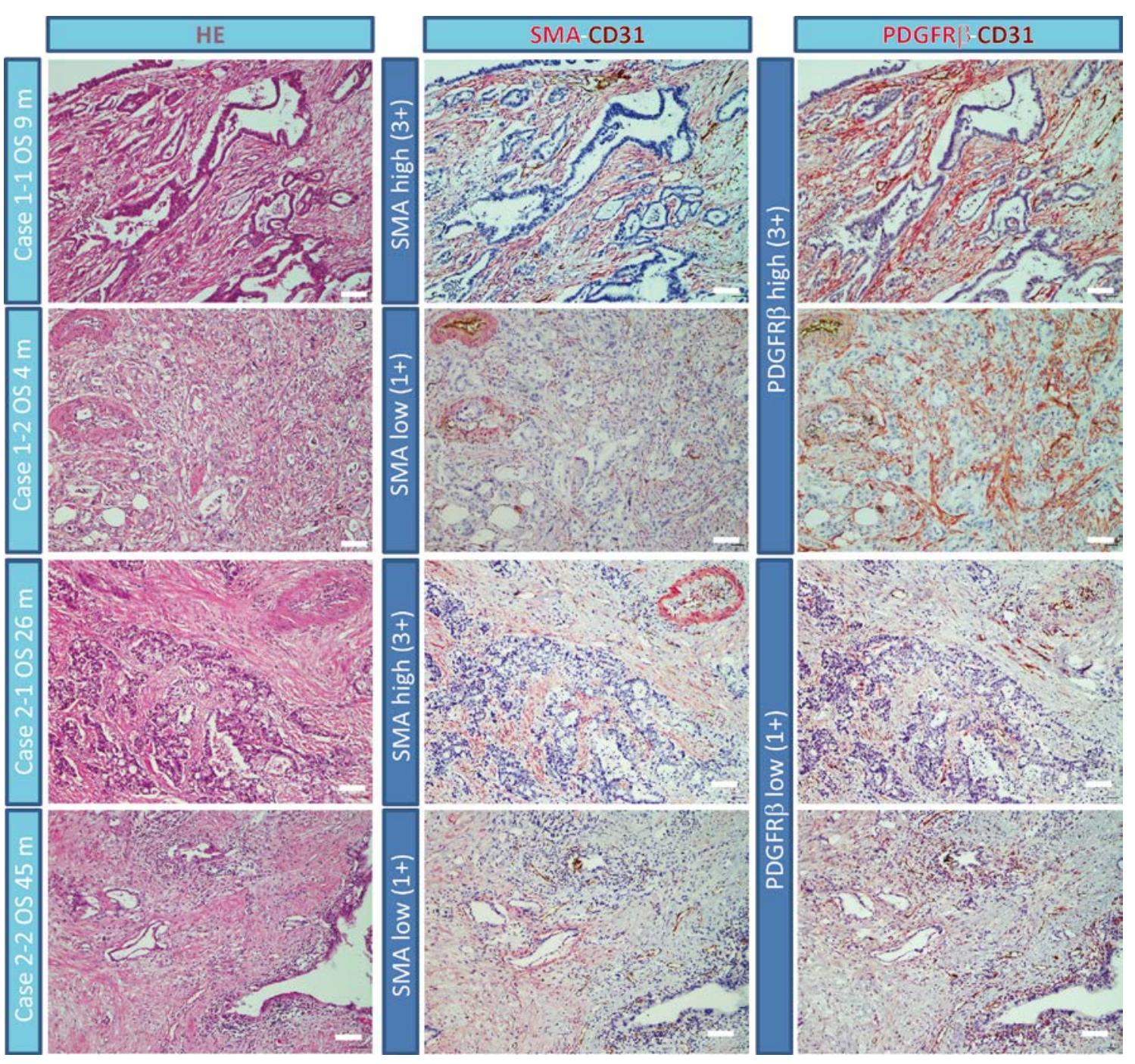


Figure 2
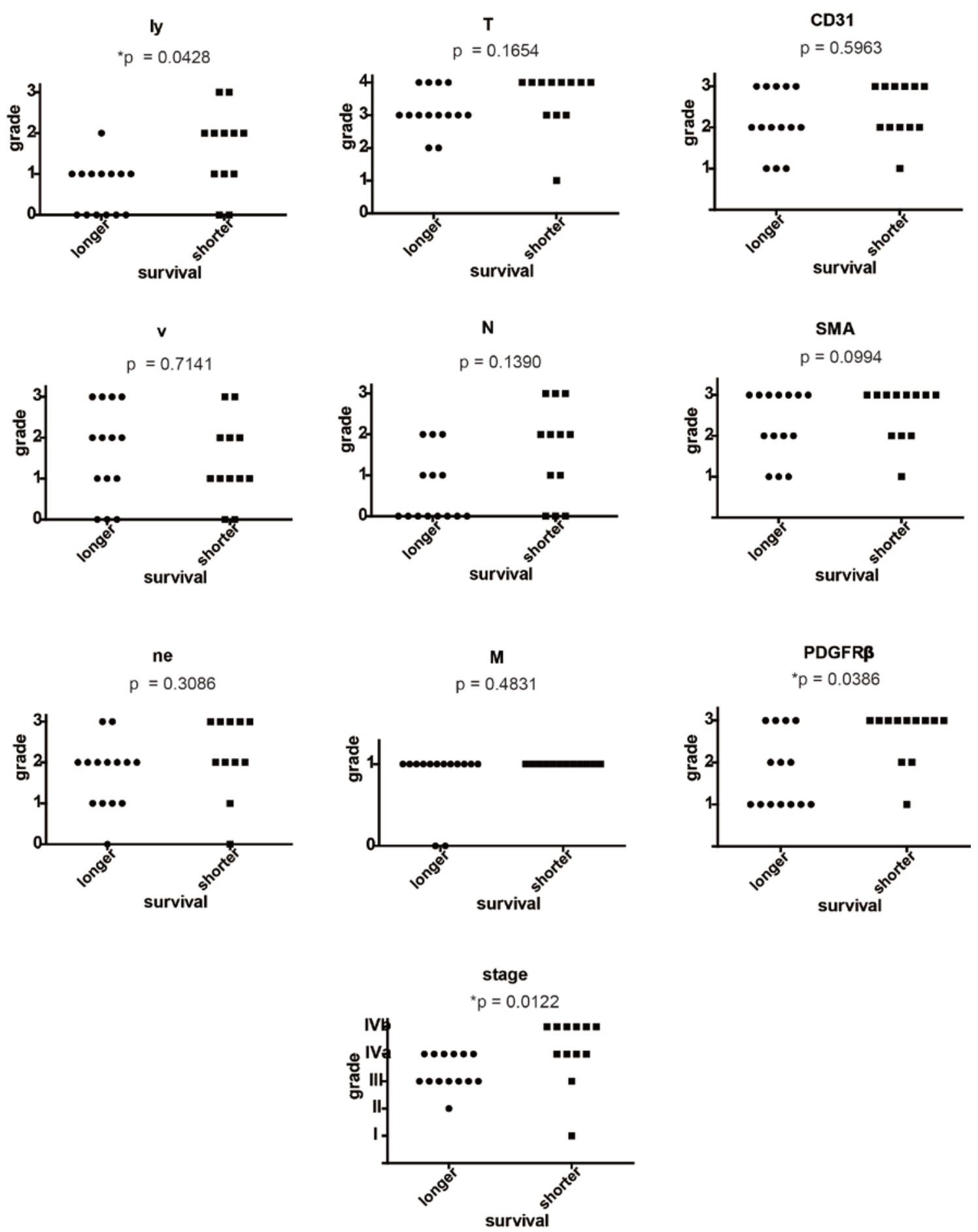
Figure 3

A

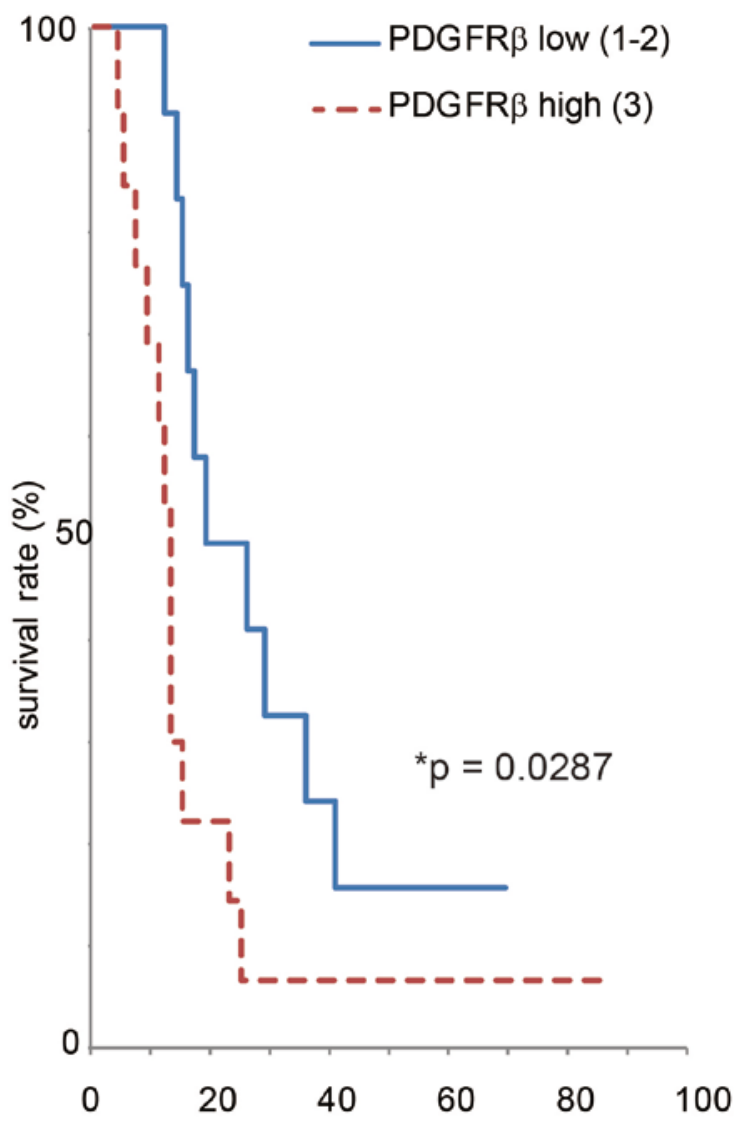

overall survival (month)
B

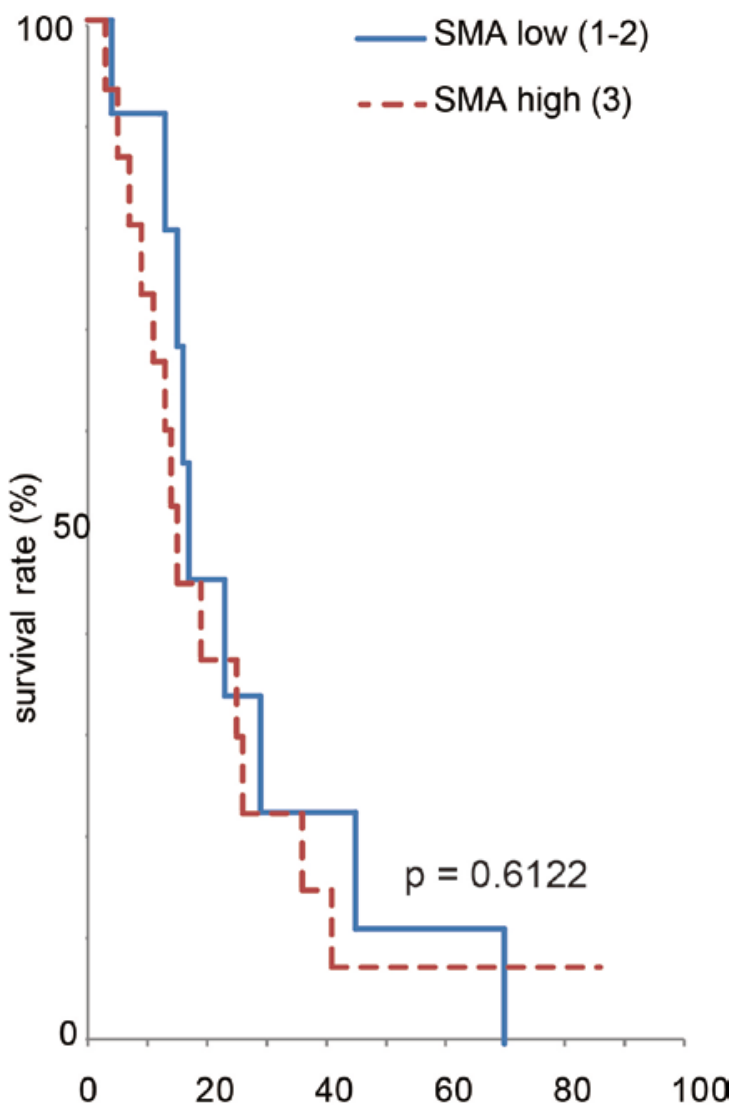

overall survival (month) 